\title{
ANÁLISIS DE LOS ENFOQUES DE APRENDIZAJE EN ESTUDIANTES DE JORNADA NOCTURNA EN RELACIÓN CON ACTIVIDADES LÚDICAS Y RECREATIVAS BASADAS EN EL JUEGO*
}

\author{
ANALYSIS OF LEARNING APPROACHES RELATED TO LEISURE AND RECREATIONAL \\ ACTIVITIES BASED ON GAME IN NIGHT-SHIFT STUDENTS
}

Manuel Guillermo Soler Contreras**

Luz Ángela Romero Vanegas***

\section{Resumen}

Se reportan resultados de una experiencia a partir de un trabajo lúdico y recreativo, basado en el juego, dirigido por estudiantes del ciclo 302 de la jornada nocturna de una institución educativa, orientados por los docentes investigadores, e impartido a los compañeros de los demás ciclos. Estos conducen a concluir que los estudiantes del ciclo 302, impulsados por una motivación intrínseca, han guiado su aprendizaje bajo un enfoque profundo. Los estudiantes de los otros ciclos, al verse orientados por sus compañeros, han mostrado una actitud de convivencia y de competencia que es necesario seguir cultivando.

Palabras clave: motivación intrínseca, motivación extrínseca, estrategia de aprendizaje, enfoque superficial, enfoque profundo.

Abstract

The results from an experience of a ludic and recreational study based on game directed by the 302 Cycle night shift students, oriented by teachers-researches and shared with their peers from other cycles are reported.302 cycle students were driven by an intrinsic motivation and have guided their learning process under the deep approach. Students from other cycles have replied this experience and have shown an attitude of coexistence and competition necessary to continue developing.

Keywords: intrinsic motivation, extrinsic motivation, learning strategy, surface approach, deep approach.

Fecha de recepción: 12 de diciembre de 2013

Fecha de aprobación: 16 de marzo de 2014

\footnotetext{
* Esta experiencia se ha realizado en el marco del proyecto de investigación sobre enfoques de enseñanza que adelanta el primer autor en la línea de investigación sobre Evaluación, en el énfasis de Ciencias, Acciones y Creencias del Doctorado Interinstitucional en Educación en la Universidad Pedagógica Nacional, con la valiosa colaboración de la segunda autora como especialista en educación deportiva.

** Doctorando UPN, Magíster en Didáctica de las Ciencias, Especialista en Análisis Químico Instrumental, Licenciado en Química. Docente investigador Secretaría de Educación del Municipio de Soacha. Correo electrónico: maguiso5@hotmail.com

*** Magíster en Docencia, Licenciada en Educación Física. Docente investigadora Secretaría de Educación del Municipio de Soacha. Correo electrónico: angjc29@hotmail.com
} 


\section{INTRODUCCIÓN}

Tomando los fundamentos teóricos de las escuelas de Gotemburgo (Marton y Säljö, 1976b) y Edimburgo (Entwistle, 1987) en relación con los enfoques de aprendizaje, se ha diseñado e implementado una estrategia lúdico-recreativa basada en el juego para beneficiar a la población estudiantil de la jornada nocturna de la institución educativa Compartir del municipio de Soacha en Colombia. La relevancia está en que, dada la naturaleza de esta población estudiantil (adultos mayores en su gran mayoría) y la modalidad de educación (jornada nocturna y formación por ciclos), los espacios lúdicos, recreativos y deportivos con que cuentan son prácticamente nulos.

Todo esto se sustenta con constructos teóricos como los enfoques de aprendizaje, motivaciones, estrategias, lúdica, recreación y juego; constructos desarrollados teóricamente para pisar en terreno firme a la hora de implementar la estrategia, recolectar la información pertinente $y$, fruto de su análisis, extraer conclusiones que den cuenta de esta experiencia significativa.

Se ha aplicado al ciclo 302 el instrumento CEA, cuestionario de enfoques de aprendizaje, traducido por Fidel Antonio Cárdenas Salgado y adaptado para el contexto colombiano por el docente investigador para caracterizar la ruta de aprendizaje seguida en el desarrollo de esta experiencia, buscando luego relacionar estos resultados con los obtenidos tras su implementación.

Esta experiencia se empezó a planificar en febrero de 2013, su implementación se dio en mayo, el análisis de los resultados y la redacción del documento, entre mayo y junio de 2013. Se proyecta continuar el proceso aprovechando los excelentes resultados obtenidos.

\section{OBJETIVOS DE LA EXPERIENCIA SIGNIFICATIVA}

Objetivo 1. Caracterizar el enfoque de aprendizaje de los estudiantes que lideran actividades lúdicas y recreativas basadas en el juego en la jornada nocturna.

Objetivo 2. Integrar a los estudiantes de la jornada nocturna a través de actividades lúdicas y recreativas basadas en el juego.

Objetivo 3. Trabajar los patrones básicos de movimiento y las capacidades físicas en estudiantes de la jornada nocturna.
Objetivo 4. Reforzar los valores de respeto y tolerancia en esta población diversa y embebida en una problemática social compleja.

\section{ASPECTOS TEÓRICOS}

Desde el punto de vista del proceso de aprendizaje, se han tomado como referentes de primera línea los planteamientos de investigadores españoles como Recio y Cabero (2008) y Hernandez Pina (1993), quienes han adoptado la línea de investigación denominada Student Aproaches to Learning (SAL) para guiar sus estudios, los constructos teóricos han surgido de las escuelas de Edimburgo, a la cabeza de Entwistle, Hanley, Hounsell y Ramsden en Inglaterra (Entwistle, 1987), y Gotemburgo, liderados por Marton y Säljö en Suecia (1976a y 1976b), y su continuación por John Biggs en Hong Kong y Australia, en relación con los enfoques de aprendizaje.

Biggs define los enfoques como "los procesos de aprendizaje que emergen de las percepciones que los estudiantes tienen de las tareas académicas, influidas por sus características de tipo personal" (Biggs, 2005), es decir, el significado no se transmite mediante la enseñanza directa que se crea mediante las actividades de aprendizaje diseñadas para tal fin.

En la línea de investigación SAL, se han identificado dos tipos de enfoques de aprendizaje: superficial y profundo. Estos enfoques están en función tanto de las características individuales de los alumnos como del contexto de enseñanza en que se da el proceso. Por ello, el enfoque de aprendizaje describe la naturaleza de la relación entre contexto, alumno, y tarea (Soler, 2012). 


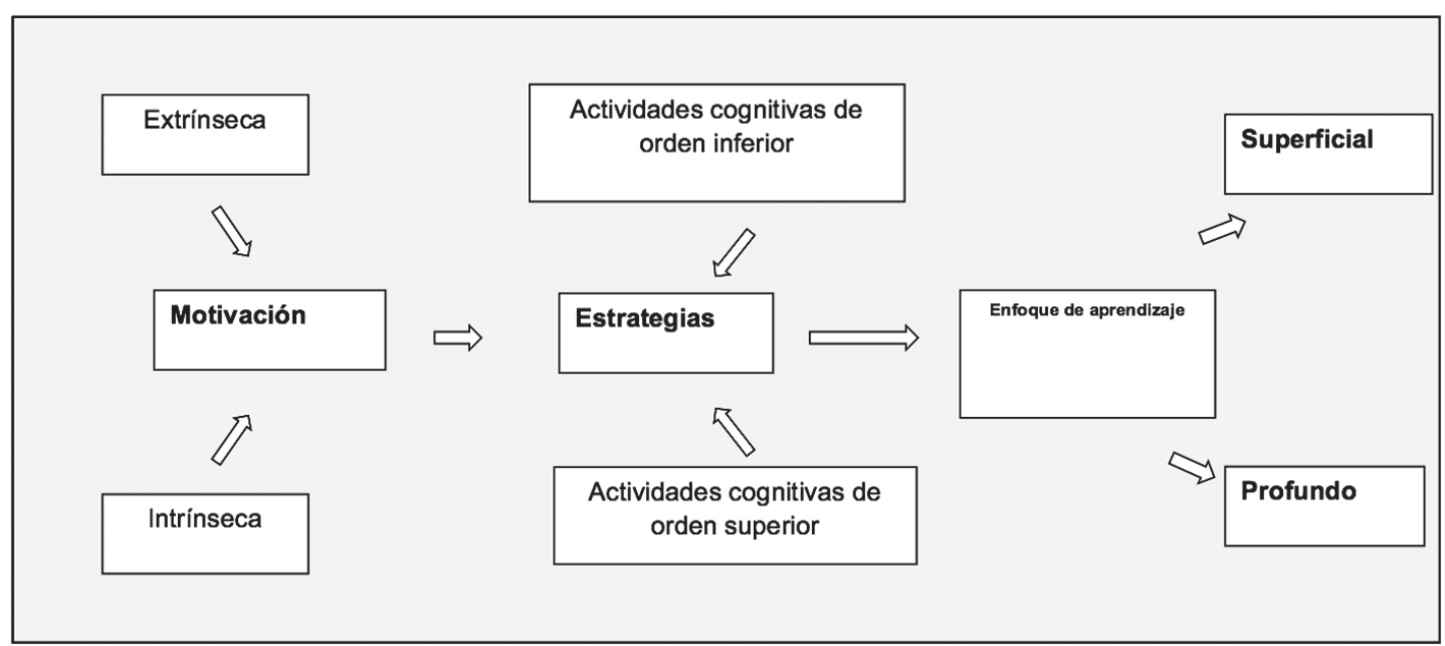

Figura 1. Ilustración de las relaciones entre los factores que intervienen en la selección del enfoque de aprendizaje por parte de cada estudiante.

En el sistema representado en la figura 1, se muestran las variables motivación y estrategias de estudio como determinantes a la hora de elegir el enfoque de aprendizaje; así, se aprecia en la parte superior del sistema que una motivación extrínseca proveniente del entorno exterior al sujeto, provoca una orientación general que hace ver la tarea académica como una imposición, la tarea se realiza con el mínimo esfuerzo y está guiada por el miedo al fracaso; esto conduce al uso de estrategias cognitivas de orden inferior tendientes a la memorización de información para reproducirla cuando sea evaluado, lo cual, a su vez, hace ver los elementos desligados de los contenidos estudiados con anterioridad, sin intentar nunca integrarlos y comprenderlos, generando sentimientos negativos de autoeficacia, esta es la ruta de aproximación al conocimiento conocida como enfoque superficial de aprendizaje.

De otro lado, la parte inferior del sistema representado en la figura 1 deja ver que una motivación intrínseca originada desde el interior del sujeto provoca en él una orientación general hacia la comprensión del significado de lo que se aprende, así la tarea se hace algo interesante con importantes implicaciones personales para cada estudiante, esto, a su vez, conduce al uso de un pensamiento de orden superior tendiente a discutir, reflexionar, teorizary plantear hipótesis; de esta manera, los elementos estudiados se ven desde una perspectiva holística que buscan una relación de los conceptos, con los contenidos de otras asignaturas, generando así en el sujeto sentimientos positivos de autoeficacia. Esta es la ruta de aproximación al conocimiento conocida como enfoque profundo de aprendizaje.
Desde la perspectiva de los enfoques de aprendizaje, el trabajo que aquí se presenta pretende encaminar a los estudiantes del ciclo 302 para que por medio de procesos autónomos elijan una ruta de aprendizaje orientada bajo el enfoque profundo, es decir, generarles una motivación intrínseca que los lleve a plantear estrategias basadas en la autorregulación para diseñar e implementar actividades lúdicas, recreativas basadas en el juego a sus compañeros de jornada, de modo que les fortalezca competencias procedimentales y actitudinales que sirvan de modelo a seguir.

En cuanto a la lúdica y la recreación, debe entenderse el juego como actividad lúdica, fuente de placer, diversión y alegría que por lo general es exaltada por quien la realiza. Se lleva a cabo de manera espontánea, voluntaria y libre, debido a que no admite imposiciones externas.

Las características del juego son las que han permitido abordarlo como medio para la creación de hábitos que den a los estudiantes las herramientas para vivir en una comunidad en gran parte científica y tecnológica en la cual se siguen reglas, normas, libertad, autonomía y responsabilidad en los espacios de convivencia que se crean con los demás. Estudios sobre la lúdica recalcan la importancia de jugar con objetos e ideas como parte del proceso de aprendizaje. El juego es en realidad un asunto serio en la educación de adultos, se persigue aquello que Palacios (2005) resalta en cuanto a que "lleva al desarrollo de habilidades de observación y experimentación y a la comprobación de ideas; ofrece la oportunidad de descubrir por uno mismo la belleza de la naturaleza". (s.p.) 
El juego debe ser visto como una actividad de disfrute donde se use adecuadamente el tiempo y la energía para que el estudiante actúe y se desenvuelva con sus propias normas y reglas que por voluntad propia acepta y cumple. El juego implica ser y hacer, por lo que requiere de la participación activa e integral de quien lo aborda.

El juego guarda conexiones sistemáticas y dinámicas con aquello que la persona debe hacer realidad y que no es considerado como juego. Mostrando su carácter holístico por estar vinculado a acciones del desarrollo humano como la creatividad, la solución de problemas, el desarrollo de lenguajes, la determinación del rol social, el aprendizaje, la comunicación, entre otras. Asimismo, el juego incrementa el deseo inmerso impulsador de toda actividad lúdica, proporcionar a los participantes una serie de capacidades, funciones y privilegios que no tienen, pero que desean.

También ofrece a quién juega la posibilidad de expresarse descubriendo su personalidad integral, permitiéndose explorar y experimentar a partir de sensaciones, movimientos y de la interacción con los demás y con la realidad exterior para reestructurar de forma progresiva su aprendizaje sobre el mundo. Gracias a esta perspectiva, se ha vivenciado el juego como estrategia que posibilita un proceso educativo, en el que se involucran mucho más tanto los que aprenden como quienes enseñan.

De igual modo, se ha encontrado que la adaptación, invención y socialización de los contenidos de los juegos permite que los educandos desarrollen e incrementen acciones como relacionar, describir, crear alternativas, comprender, establecer razones para validar o refutar, escuchar, dialogar, negociar, aceptar, diferenciar, entre otras. Estas acciones, a su vez, han estado involucradas de forma directa en el proceso comunicativo-científico, a través del cual se ha evidenciado el desarrollo de competencias comunicativas de tipo discursivo como interpretar, argumentar y proponer.

Para alcanzar un aprendizaje significativo y lograr una educación con carácter científico, se debe trabajar el juego no solamente como una actividad espontánea, sino que se debe analizar su dirección y orientación pedagógica. Es decir, se debe lograr que la actividad del juego tenga un carácter organizado, tendiente a activar pensamientos rápidos y coherentes con los objetivos y contenidos de la enseñanza, buscando promover de forma eficiente el aprendizaje y satisfacer las necesidades y el placer de los alumnos (Concepción, 2004).

La posibilidad de asimilar el juego como estrategia se ha dado gracias a que tiene un valor didáctico, que combina aspectos para organizar de manera eficiente el proceso de enseñanza y el aprendizaje como la participación, el dinamismo, el entrenamiento, la interpretación de papeles, la colectividad, la modelación, el carácter problémico, la retroalimentación, la obtención de resultados, la iniciativa, el carácter sistémico y la competencia (Bautista y López, 2002).

Vale la pena hacer énfasis en que el desempeño comunicativo en grupo permite que los estudiantes compartan sus conocimientos para enseñar y aprender de sus pares y el profesor, debido a que se plantean problemas y preguntas relativas a las temáticas, promoviendo el desarrollo intelectual y despertando una intensa y profunda motivación por el estudio (González, 2003). Es por esta razón que se puede trabajar más en situaciones específicas a través de actividades lúdicas que mantengan al estudiante en alerta todo el tiempo (porque el estudiante debe emplear adecuadamente los contenidos temáticos, para poder crear o adaptar los juegos, así como para jugarlos) para adecuar espacios en los cuales se pueda desempeñar de manera amena y al mismo tiempo impedir que los ejes temáticos se tornen aburridores y difíciles de comprender.

Estas situaciones también facilitan a los estudiantes y docentes-investigadores mostrarse como creadores de ideas nuevas, con las cuales expresan capacidades que no pensaron tener. Y los motivan y animan para elevar su autoestima y permiten que entiendan como utilizar y compartir dichas capacidades. La posibilidad de motivar despierta, mantiene y refuerza la conducta para que se perciban a sí mismos como seres capaces de lograr metas. Esta motivación se afianza aún más si se le asigna al estudiante el diseño de su propio juego o material lúdico, como ha ocurrido en la experiencia que aquí se comparte.

Asimismo, se ha encontrado que a través del juego, la persona que enseña puede descubrir la capacidad que poseen sus estudiantes para construir conceptos abstractos, al mismo tiempo que ellos pueden encontrar las reglas intrínsecas que hacen coherente aquello que están aprendiendo. Gracias a que el juego y la creatividad son procesos muy relacionados, las personas vivencian sus experiencias lúdicas con una concentra- 
ción y absorción total. La opción de jugar y crear ofrece a cada individuo la posibilidad de involucrarse en un proceso de elección, a través del cual puede y debe asumir responsabilidades que lo conducen a actualizar sus potencialidades, para ser tan bueno(a) como pueda, atreverse, expresarse sinceramente; llegando a descubrir quién y cómo es realmente (Jiménez, 1998).

Aunque el juego y la lúdica no son cualidades ni dones, sí son dimensiones del desarrollo humano propias de sus comportamientos culturales y biológicos. En este sentido, puede considerarse el juego como un fenómeno biológico y cultural, propio de ámbitos comunicativos que contribuyen al desarrollo humano en sus espacios de creatividad y convivencia (Jiménez, 2000). La idea entonces consiste en generar una disciplina consciente, desde la cual se asimilen las pautas comunicativas que rigen el logro del aprendizaje y, a partir de esto, buscar que se conciba y experimente la necesidad de cumplir con sus normas y compromisos (González, 2003).

\section{METODOLOGÍA}

La experiencia consiste en realizar, bajo la modalidad de la investigación-acción (Elliott, 1990), una práctica lúdica, recreativa y deportiva basada en el juego con los estudiantes de la jornada nocturna, que, orientada por los estudiantes de uno de los ciclos y bajo la tutela de los docentes investigadores, se imparte a los estudiantes de los demás ciclos de esta jornada con el propósito de afianzar aspectos conceptuales relativos a la habilidades motoras y organizacionales, en los primeros, por medio de estrategias metacognitivas, y de fomentar habilidades procedimentales y actitudinales en los segundos. Como grupo organizador, se han escogido los estudiantes del ciclo 302 de la institución educativa Compartir del municipio de Soacha (Colombia), quienes son personas que trabajan, a la vez que son padres y madres de familia, y como valor agregado quieren estudiar.

A continuación (tabla 1) se muestran las características del grupo que lidera la experiencia.

Tabla 1. Descripción del grupo que lidera la experiencia significativa.

\begin{tabular}{|c|c|}
\hline \multicolumn{2}{|c|}{ Caracterización del grupo } \\
\hline Estudiantes & Ciclo III \\
\hline Mujeres & 9 \\
\hline Hombres & 7 \\
\hline Total & 16 \\
\hline Edad & Entre $18 \mathrm{y} 47$ años \\
\hline
\end{tabular}

Es de resaltar la variabilidad de edades, pues entre los extremos 18 y 47 hay todo un espectro de edades que le proporciona una naturaleza bastante heterogénea a este grupo.

Se ha seleccionado, entre muchas opciones, un trabajo lúdico, recreativo y deportivo basado en el juego, dada su sencillez en cuanto a materiales usados, al procedimiento a seguir y a la necesidad de este tipo de actividades en esta población estudiantil.

La experiencia significativa se da en cuatro momentos:

- En el primer momento, se determina que la actividad a realizar es un circuito ${ }^{1}$, actividad que permite involucrar a todos los estudiantes de la jornada nocturna, y además se establecen los juegos y se eligen los representantes (coordinador general y un coordinador de material).

- En el segundo momento, se establece que se realizarán 16 estaciones, asignándole a cada estudiante del ciclo 302 una de ellas, para que este elabore un juego, el cual debe estar relacionado con los objetivos y debe contener las siguientes características: nombre, debe ser creativo y que se pueda valorar asignando puntos.

- En el tercer momento, se diseña un plano de la institución para la ubicación de las estaciones (anexo 1) y se establece el tiempo a emplear (80 minutos en total haciendo la rotación de estación cada cinco minutos).

- Como cuarto y último momento, se le asigna a cada docente de la jornada nocturna una estación y se le dan las instrucciones pertinentes.

\section{RESULTADOS}

En este apartado, se consignan los resultados en coherencia con los objetivos, inicialmente planteados así:

Objetivo 1. Caracterizar el enfoque de aprendizaje de los estudiantes que lideran actividades lúdicas y recreativas basadas en el juego en la jornada nocturna

Tras aplicar el instrumento CEA a los alumnos del ciclo 302 con la intención de identificar el enfoque de aprendizaje predominante usado en esta experiencia, se ha tabulado y graficado la información que se muestra a continuación (tabla 2).

1 Los circuitos se desprenden del entrenamiento deportivo, lo cual consiste en realizar una serie de ejercicios o actividades recreativas, las cuales tiene por nombre estaciones o bases llevando un orden consecutivo. 
Tabla 2. Puntuación de los enfoques superficial y profundo, tipo e intensidad del enfoque de los estudiantes del ciclo 302.

\begin{tabular}{|c|c|c|c|c|c|}
\hline $\begin{array}{l}\text { Cod. } \\
\text { Est. }\end{array}$ & \begin{tabular}{|l} 
Enfoque \\
Profundo
\end{tabular} & $\begin{array}{l}\text { Enfoque } \\
\text { Superficial }\end{array}$ & $\begin{array}{l}\text { Diferencia } \\
\text { entre Ios } \\
\text { Puntajes de } \\
\text { los } \\
\text { Enfoques }\end{array}$ & \begin{tabular}{|l|} 
Tipo de \\
Enfoque
\end{tabular} & $\begin{array}{l}\text { Intensidad } \\
\text { del Enfoque }\end{array}$ \\
\hline 1 & 38 & 38 & $\mathbf{0}$ & Intermedio & Débil \\
\hline 2 & 28 & 31 & -3 & Superficial & Débil \\
\hline 3 & 45 & 32 & 13 & Profundo & Moderado \\
\hline 4 & 45 & 45 & $\mathbf{0}$ & Intermedio & Débil \\
\hline 5 & 43 & 39 & 4 & Profundo & Débil \\
\hline 6 & 44 & 47 & -3 & Superficial & Débil \\
\hline 7 & 50 & 51 & -1 & Superficial & Débil \\
\hline 8 & 55 & 23 & 32 & Profundo & Fuerte \\
\hline 9 & 43 & 40 & 3 & Profundo & Débil \\
\hline 10 & 49 & 29 & 20 & Profundo & Fuerte \\
\hline 11 & 21 & 25 & -4 & Superficial & Débil \\
\hline 12 & 34 & 42 & -8 & Superficial & Moderado \\
\hline 13 & 48 & 41 & 7 & Profundo & Moderado \\
\hline 14 & 52 & 42 & 10 & Profundo & Moderado \\
\hline 15 & 39 & 39 & 0 & Intermedio & Débil \\
\hline 16 & 42 & 47 & -5 & Superficial & Débil \\
\hline
\end{tabular}

La tabla 2 muestra en las columnas 2 y 3 la puntuación que cada estudiante ha obtenido para los enfoques superficial y profundo, respectivamente; la columna 4 corresponde a la diferencia entre las columnas antes mencionadas, un valor positivo indica un enfoque profundo y un puntaje negativo, un enfoque superficial, esto se evidencia en la columna 5; la columna 6, correspondiente a la intensidad del enfoque, se interpreta haciendo uso de unos baremos que definen el enfoque como débil, moderado o fuerte, así: valores entre 0 y \pm 7 corresponden a una intensidad débil; valores entre 8 $\mathrm{y} \pm 14$, a una intensidad moderada y valores entre $15 \mathrm{y}$ \pm 20 , a una intensidad fuerte.

A continuación se presenta la interpretación de los datos reportados en la tabla 2 (gráfica 1 ).

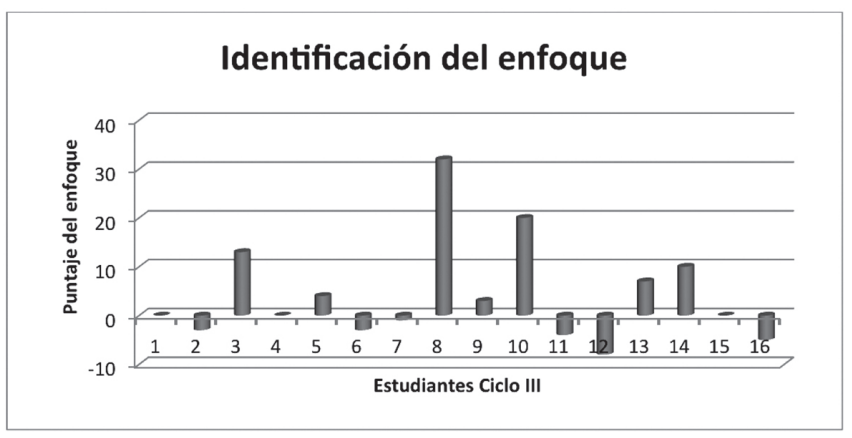

Gráfica 1. Caracterización del enfoque de aprendizaje en los estudiantes del ciclo 302.
Las barras con orientación vertical hacia arriba del eje de base en la figura 1 corresponden a estudiantes cuyas rutas de aprendizaje adoptadas se orientan bajo un enfoque profundo (estudiantes con códigos 3, 5, 8, 9, 10,13 y 14), lo que equivale a un $43,7 \%$ del grupo estudiado; la barras con orientación vertical hacia abajo del eje de base corresponden a estudiantes cuyas rutas de aprendizaje adoptadas se orientan bajo un enfoque superficial (estudiantes con códigos 2, 6, 7, 11, 12 y 16 ), lo que equivale a un $31,2 \%$ del grupo estudiado, y los puntos que no presentan ninguna orientación vertical corresponden a estudiantes cuyas rutas de aprendizaje adoptadas son justamente intermedias entre los dos enfoques (estudiantes con códigos 1, 4 y 15), para un $18,7 \%$ del grupo estudiado.

Objetivo 2. Integrar a los estudiantes de la jornada nocturna a través de actividades lúdicas y recreativas basadas en el juego

En cuando al grado de convocatoria y participación logrado por estudiantes del ciclo 302 en sus demás compañeros de la jornada nocturna, esta fue del 100\%; en la logística, los estudiantes del ciclo 302 involucraron a todos sus profesores, al personal administrativo y de servicios generales, quienes cumplieron a cabalidad las funciones asignadas, garantizando la total participación de todos los estudiantes de la jornada (anexo 2).

Objetivo 3. Trabajar los patrones básicos de movimiento y las capacidades físicas en estudiantes de la jornada nocturna

Los patrones básicos de movimiento, como desplazamientos, saltos, giros y manipulaciones, han sido trabajados en las diferentes estaciones. Se ha puesto en evidencia que un alto número de participantes no tiene dominio de estos patrones, las principales dificultades detectadas están en los saltos y giros en estudiantes que pertenecen a la población adulto-mayor. En cuanto a las capacidades físicas como fuerza, agilidad, equilibrio, coordinación, han sido estas dos últimas en las que se ha presentado mayor dificultad en su desempeño.

Objetivo 4. Reforzar los valores de respeto y tolerancia en esta población diversa y embebida en una problemática social compleja 
Se ha generado un ambiente competitivo pero con total tolerancia y respeto por el otro, comportamiento poco común en esta población conflictiva por naturaleza. Se han identificado en cada grupo unos líderes que espontáneamente se han puesto al comando de su equipo. Las funciones asignadas por el ciclo 302 a los docentes de la jornada, al personal administrativo y de servicios generales han sido cumplidas con sentido de pertenencia y han manifestado haber disfrutado del proceso.

\section{ANÁLISIS DE RESULTADOS}

Los datos relacionados en el apartado anterior muestran que el enfoque predominante de aprendizaje en esta experiencia ha sido el profundo, seguido por el superficial y el intermedio, respectivamente. Sin embargo, la intensidad del enfoque superficial es bastante baja, quedando en realidad muy cerca del enfoque intermedio. Por lo que se ve, hay una clara tendencia hacia el uso de una ruta profunda de aprendizaje (con elevadas intensidades en algunos casos) en este tipo de actividades lúdico-recreativas y deportivas. Esto se encuentra en coherencia con el nivel de compromiso que estos estudiantes lograron despertar en sus demás compañeros de la jornada nocturna, quienes realizaron todo el circuito prestando máximo interés y asumiendo diversos roles de acuerdo con los retos que se les presentó en cada estación.

Las expectativas en cuanto a la puesta en práctica de valores como el respeto y la tolerancia fueron de lejos superadas, pues el comportamiento ha sido atípicamente excelente, no se presentó ningún tipo de altercados de los que suelen presentarse en el aula o en el descanso en las jornadas habituales.

Se ha puesto de manifiesto cómo el sedentarismo y la falta de realización de actividad física en este tipo de población estudiantil contribuyen a la pérdida de habilidades de dominio de los patrones básicos de movimiento y de capacidades físicas.

\section{CONCLUSIONES}

Este tipo de actividades, poco comunes en esta modalidad de educación para adultos en la jornada nocturna, promueve desempeños que suelen permanecer ocultos en las actividades académicas cotidianas, se resalta la autonomía, la autorregulación y el uso de estrategias metacognitivas utilizadas en el diseño e implementación de la experiencia significativa implementada.

En relación con los enfoques de aprendizaje utilizados por el grupo líder, es de resaltar cómo una motivación intrínseca en los integrantes del ciclo 302 ha permitido el desarrollo de estrategias apropiadas para este contexto, garantizando el éxito de la experiencia significativa, lo que guarda coherencia con el enfoque profundo predominante, identificado mediante el instrumento CEA. Lo anterior comprueba la dependencia del contexto y de la naturaleza de la tarea en la adopción del enfoque de aprendizaje, pues en esta población, por múltiples razones, ya sean de tiempo, de conocimientos previos deficientes o de una abundante cobertura curricular con escasa profundidad, lo que se ve es que predomina un enfoque superficial a la hora de encarar los procesos de aprendizaje.

De otro lado, la jornada nocturna cuenta con una población muy diversa, estas actividades permiten hacer un reconocimiento del otro y de sus capacidades, haciendo, además, que se profundice en valores como el respeto, la tolerancia y se trabaje la igualdad, dejando de lado la discriminación por la edad, el género, los gustos y la condición socio-económica, entre otros. Es así que esta actividad ha trabajado los patrones básicos de movimiento (locomotor, no locomotor y manipulativos) y las capacidades físicas (coordinación, fuerza, agilidad, equilibrio), que, debido a las múltiples ocupaciones de los participantes, no se practican, por lo que el sedentarismo es una constante.

Finalmente, ha quedado evidente que la lúdica y la recreación fundamentadas en el juego se muestran como una potente forma de trabajar los valores en los estudiantes de la jornada nocturna, siendo también una manera de fortalecer la autonomía, el compromiso y el sentido de pertenencia por la institución. 


\section{REFERENCIAS BIBLIOGRÁFICAS}

Bautista, J. y López, N. (2002). El juego didáctico como estrategia de atención a la diversidad. Agora Digital. Revista Científica Electrónica, 4, (3). Recuperado de http://dialnet.unirioja.es/servlet/revista?tipo busqueda $=$ CODIGO\&clave_revista $=4272$.

Biggs, J. (2005). Calidad del aprendizaje universitario. Madrid: Nacea.

Concepción, J. (2004). Estrategia didáctica lúdica para estimular el desarrollo de la competencia comunicativa en idioma Inglés de estudiantes de especialidades biomédicas. (Tesis presentada en opción al grado científico de doctor en ciencias pedagógicas). Universidad Central Marta Abreu de las Villas, Centro de Estudio de Educación Superior, Facultad de Educación a Distancia. Santa Clara, Villa Clara, Cuba. Recuperado de http://www.sld.cu/galerias/pdf/ sitios/revsalud/tesis_jose-a._concepcion.pdf.

Elliott, J. (1990). ¿Por qué deben investigar los profesores? En J. Elliott (s. f.), La investigación-acción en educación (pp. 176-190). Madrid: Morata.

Entwistle, N. (1987). La comprensión del aprendizaje en el aula. Madrid: Paidós.

González, D. (2003). La motivación: varita mágica de la enseñanza y la educación. Revista Magisterio. Educación y Pedagogía, (5), 46-49.

Hernandez Pina, F. (1993). Concepciones en el estudio del aprendizaje de los estudiantes universitarios. Revista de Investigación Educativa, (22).

Jiménez, C. A. (1998). Pedagogía de la creatividad y de la lúdica. Bogotá: Cooperativa Editorial Magisterio.

Kember, D., y Gow, L. (1993). Conceptions of teaching an teir relationship to student learning. British Journal of Educational Psychology, (63), 20-23.
Marton , F. y Säljö, R. (1976a). On qualitative differences in learning: 1. Outcome and process. British Journal of Educational Psychology, 46, 4-11.

Marton, F., y Säljö, R. (1976b). On quialitative differences in learning: 2. Outcome as a function of the leamer's conception of the task. British Journal of Educational Psychology, 115-217.

Palacios, N. (2005). La ciencia al alcance de todos: educación científica a través del juego y la diversión. Revista Magisterio. Educación y Pedagogía, 16, 74-77.

Porto, A. (1994). Las aproximaciones al proceso de aprendizaje en estudiantes universitarios. (Disertación doctoral, no publicado). Santiago de Compostela.

Prosser, M. y Trugwell, K. (2006). Conformatory factor analysis of the approaches to teaching inventory. British Journal of educational Psychology, 76, 405-419.

Ramsden, P. (1992). Learning to teach in Higher Education. Londres: Routlege.

Recio S., M., y Cabero, A..J. (2005). Enfoques de aprendizaje, rendimiento académico y satisfacción de los alumnos en formación en entornos virtuales. Pixel-Bit Revista de Medios y Educación, 25, 93-115.

Soler, C. M. (2012). El pensamiento de John Biggs. Algunos elementos teóricos acerca del alineamiento constructivo y los enfoques de aprendizaje. Memorias III congreso internacional de investigación en educación, pedagogía y formación docente.

Trigwell, K., Prosser, M. y Ginns, P. (2005). Phenomenographic pedagogy and a revised approaches to Teaching Inventory. Higher Education Research \& Development, 24, (4), 349-360.

Trigwell, K., Prosser, M. y Waterhouse, F. (1999). Relations between teachers'approaches to teaching and students'approaches to learning. Higher Education, $37,57-70$ 


\section{ANEXO 1.}

Distribución de las estaciones en el plano de la institución

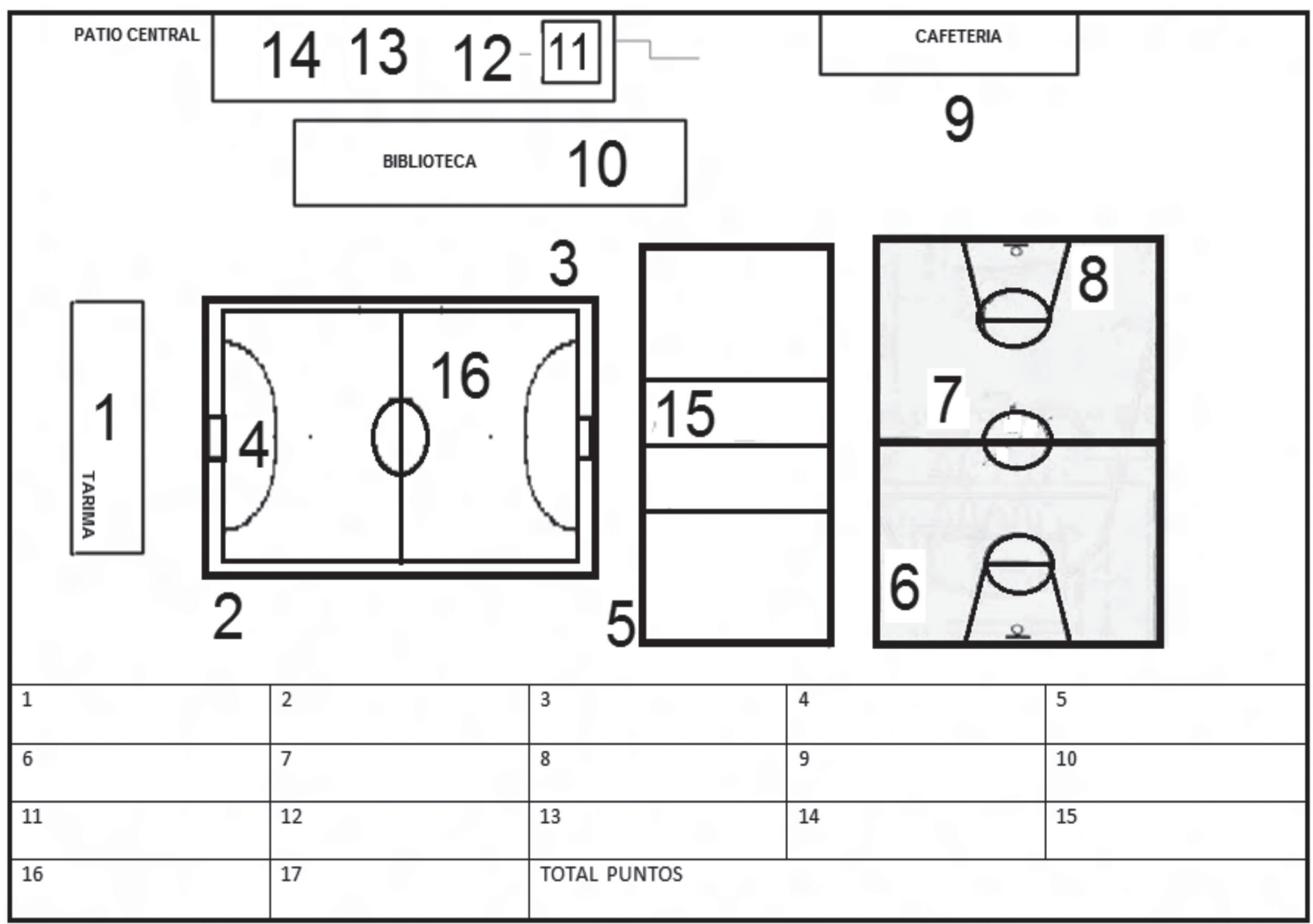

ANEXO 2.

Estaciones del circuito en acción

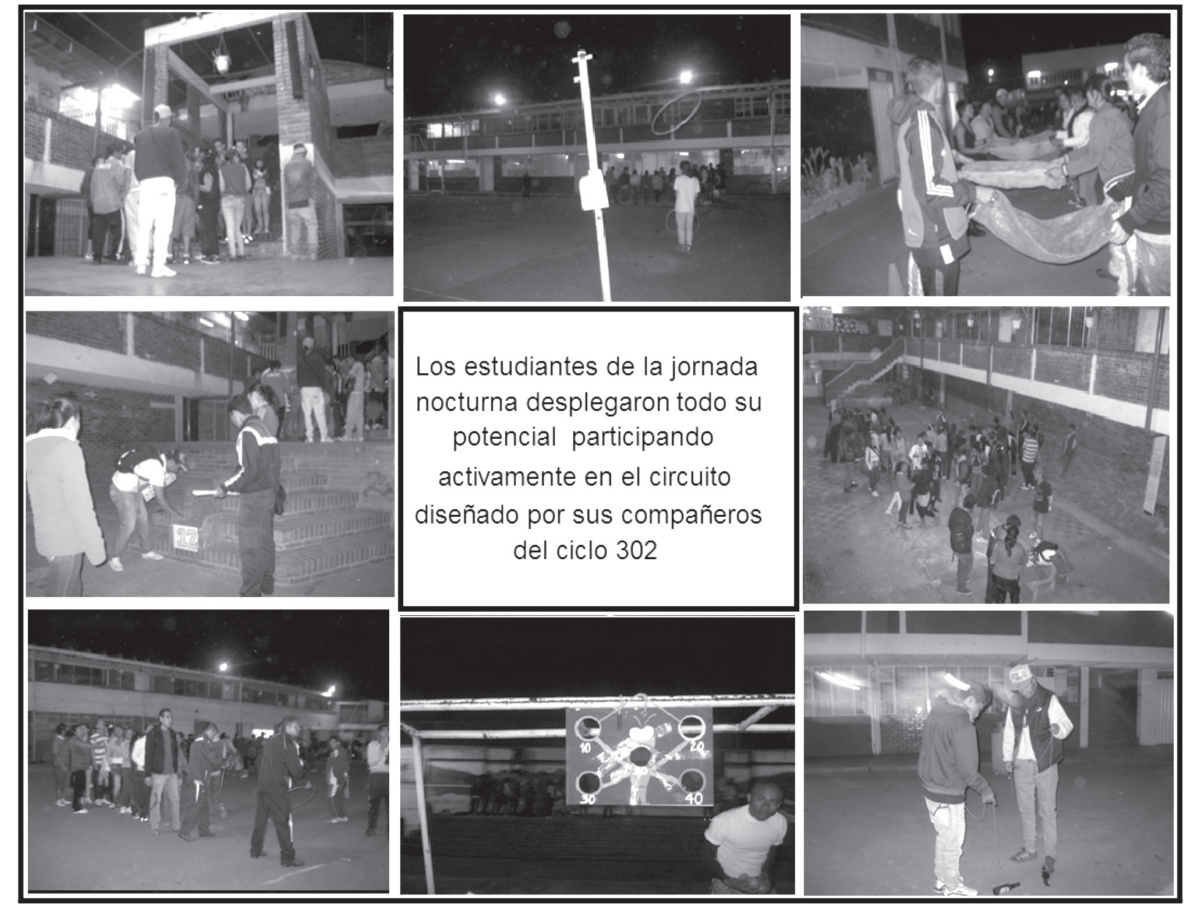

